\title{
The intestinal flora of patients with GHPA affects the growth and the expression of PD-L1 of tumor
}

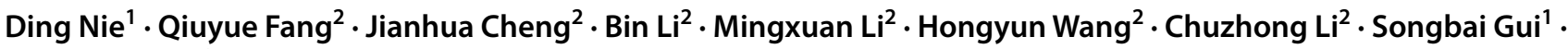 \\ Yazhuo Zhang ${ }^{2}$. Peng Zhao' ${ }^{10}$
}

Received: 13 June 2021 / Accepted: 1 October 2021 / Published online: 13 October 2021

(c) The Author(s) 2021

\begin{abstract}
Context Pituitary adenoma (PA) is a common intracranial tumor. The evidence indicates that the tumor immune microenvironment (TIME) is associated with PA and that the intestinal flora influences other tumors' growth through interacting with the TIME. However, how the intestinal microbial flora contributes to the development of PA through the immune response is unknown.

Objective and methods Here we used high-throughput Illumina MiSeq sequencing targeting the V3-V4 region of the 16S ribosomal RNA gene to investigate the intestinal flora of patients with growth hormone-secreting pituitary adenoma (GHPA), nonfunctional pituitary adenoma (NFPA), and healthy controls. We determined their effects on tumor growth and the TIME. Fecal microbiota transplantation (FMT) was performed after adoptive transfer via peripheral blood mononuclear cells to tumor-bearing nude mice, which allowed the study of the immune response.

Result We discovered differences in the structures and quantities of intestinal flora between patients with GHPA, patients with NFPA, and healthy controls. After FMT, the intestinal flora of GHPA patients promoted the growth of tumors in mouse models. The number of programmed cell death ligand 1 (PD-L1)-positive cells increased in tumor tissues as well as the extent of infiltration of $\mathrm{CD} 8^{+}$cells. Increased numbers of $\mathrm{CD}^{+}{ }^{+} \mathrm{CD} 8^{+}$cells and increased levels of sPD-L1 were detected in peripheral blood.

Conclusion These findings indicated that the intestinal flora of patients with GHPA promoted tumor growth and that the immune system may mediate this change.
\end{abstract}

Keywords Pituitary adenoma $\cdot$ Intestinal flora $\cdot$ Immune $\cdot$ PD-L1

\section{Introduction}

Pituitary adenoma (PA) is a common endocrine tumor of the central nervous system, which is classified according to hormone secretion as functional pituitary adenoma (FPA) and nonfunctional pituitary adenoma (NFPA) [1]. Growth hormone-secreting pituitary adenoma (GHPA) is

This study reveals the influence of intestinal flora on tumor growth in patients with pituitary adenoma and the possible role of immunity.

Peng Zhao

zhaopeng@ccmu.edu.cn

1 Department of Neurosurgery, Beijing Tiantan Hospital, Capital Medical University, Beijing, China

2 Beijing Neurosurgical Institute, Beijing, China a common subtype of FPA [2]. Excess production of GH leads to acromegaly, heart disease, sleep apnea, and other conditions that shorten life expectancy [3]. After systemic treatment (surgery and medication), certain GHPAs challenge effective clinical treatment because of relapse or drug resistance $[4,5]$. Therefore, factors affecting the occurrence and development of GHPA must be identified.

Inflammatory immune cells, chemokines, and cytokines influence tumor growth and invasion [6]. Multiple studies show that GHPAs are associated with more $\mathrm{CD} 4^{+}$cells and $\mathrm{CD}^{+}$cells than non-GH adenomas as well as increased levels of programmed death ligand 1 (PD-L1) [7-10]. PD-L1 binds to the PD- 1 receptor on activated $T$ cells and inhibits the cytotoxic antitumor function of $T$ cells [11-13]. For example, anti-PD-L1 treatment reduces plasma ACTH levels of model mice, decreases the growth of adenomas, and increases the survival rate of model mice [10]. Although 
no further study was conducted in that report, the expression of PD-L1 in GHPA was the highest in their study [10]. However, the factors affecting tumor expression of PD-L1 are unknown and must be identified to optimize immune checkpoint blockade therapy of PA.

The composition of the intestinal flora regulates the development of the central nervous system and contributes to the development of nervous system pathologies $[14,15]$. The intestinal flora is a key environmental factor that affects the immune homeostasis of the host $[16,17]$. For example, 11 distinct bacterial communities activate mouse $\mathrm{CD} 8^{+} T$ cells to effectively inhibited tumor growth [18]. Bifidobacterium species enhance the antitumor effects of PD-L1 inhibitors as well as the functions of dendritic cells and $\mathrm{CD} 8^{+}$ $T$ cell-mediated antitumor mechanisms [19]. However, to our knowledge, direct evidence does not support the conclusion that the composition of intestinal microbiota affects the development or progression of PAs. Therefore, here we sequenced the bacterial flora in stool samples of healthy controls and patients with NFPA or GHPA. We determined the immune markers of GHPA and NFPA. We used immune reductionist mice to determine the effects of FMT on tumor formation by xenotransplanted $\mathrm{GH} 3$ cells. The changes in tumor and immune indexes were observed.

\section{Materials and methods}

\section{Clinical studies and specimen processing}

All studies were conducted under the approval of the Institutional Review Committee of Beijing Tiantan Hospital, Capital Medical University, and the study was conducted by the Principles of Good Clinical Practice and the Declaration of Helsinki. 50 patients with PA (25 GHPA,25 NFPA) were recruited from Beijing Tiantan Hospital, Capital Medical University. Twenty-five healthy subjects were recruited from a qualified population in Beijing, China.

Patients, who also met pathologically confirmed PA (according to the standards of The World Health Organization (WHO) classifications of tumors), had no history of chronic digestive tract disease, no other diseases (cold, etc.) on admission, had no history of chronic metabolic disease, did not take antibiotics or microbial agents within 1 week, had no history of cardiovascular disease, malignant tumor or other diseases, and had no history of smoking or alcohol abuse were considered to meet the inclusion criteria. Patients who did not meet the inclusion criteria were excluded. Controls were matched to PA patients concerning age, sex, following the same exclusion criteria as used for PA patients (Supplementary Table).

A total of 75 fresh feces were collected preoperatively, and all the donors fasted for $12 \mathrm{~h}$ before collection. Each sample was separately collected with three sterile PV tubes in the morning, sealed and temporarily stored in $-20^{\circ} \mathrm{C}$, and transferred to $-80^{\circ} \mathrm{C}$ within $2 \mathrm{~h}$ for long-term storage. Human blood samples were collected from the 75 subjects after overnight fasting. Serum samples were centrifuged and stored at $-80^{\circ} \mathrm{C}$.

\section{DNA extraction and 16SrRNA gene amplification}

Bacterial DNA extraction and PCR amplification of the V3-V4 region of 16SrRNA gene was performed in Novogene Bioinformatics Technology Co., Ltd. The Illumina MiSeq platform was used to sequence the readings and obtain the results.

\section{Mice study}

The contents and procedures related to animal testing involved in this study have been approved by the Institutional Animal Care and Use Committee (IACUC).

Female B-NDG(NOD-Prkdc ${ }^{\text {scid }}$ IL2 $\mathrm{rg}^{\text {tm1 } 1 / B c g e n) m i c e ~}$ were purchased from Biocyto (Beijing) Co., Ltd, with the age of 7-8 weeks and the weight of 200-220 g. In the absence of specific pathogens, all mice were raised in the barrier system of Serve Accurate Faithful Evaluation Co. (temperature, $20-26{ }^{\circ} \mathrm{C}$; humidity, 40-70\%; 12-h light/12-h dark cycle) and fed a rodent diet ad libitum.

The cell line was Wistar Furth rat GH3 cells purchased from ATCC. The cell suspension with a concentration of $2.5 \times 10^{7}$ cells $/ \mathrm{mL}$ was subcutaneously injected into the back of mice near the armpit. Each mouse was inoculated with $0.2 \mathrm{~mL}$ and the inoculation dose was $5 \times 10^{6}$ cells/mouse.

\section{Immune reductionist}

Female Wistar rats were purchased from Charles River (Beijing), with the age of 7-8 weeks, and were raised in the same environment as mice.

After the rats were anesthetized with isoflurane, the abdominal cavity was opened, and all blood $(8-15 \mathrm{~mL})$ of the rats was collected through the abdominal aorta in the heparin anticoagulant tube until the rats died. PBMC of Wistar rat was extracted by gradient centrifugation using the rat peripheral blood monocyte isolation liquid kit. The PBMC was injected into B-NDG tumor-bearing mice by tail vein injection, $0.5-1 \times 10^{7}$ cells per mice. After 3 days, $0.2-0.3 \mathrm{~mL}$ of peripheral blood was collected from an orbital vein in an anticoagulant tube. $\mathrm{CD} 45^{+}$and $\mathrm{CD}^{+}$cells in the peripheral blood of mice were determined by flow cytometry to verify the results of immune remodeling. When $\mathrm{CD} 45^{+}$ cells can be detected in the peripheral blood of mice, the immune reductionist is considered to be successful, and $\mathrm{CD}^{+}$cells confirmed the presence of $T$ cells [20-22]. 


\section{Fecal microbiota transplantation}

Mice with successful immune reductionists were placed in autoclaved drinking water with a mixture of broad spectrum antibiotics ( $1 \mathrm{~g} / \mathrm{L}$ ampicillin, $1 \mathrm{~g} / \mathrm{L}$ metronidazole, $1 \mathrm{~g} / \mathrm{L}$ neomycin, and $0.5 \mathrm{~g} / \mathrm{L}$ vancomycin) in the middle. Fresh stool samples were collected from two NFPA patients, two GHPA patients, and two control patients. The $500 \mathrm{mg}$ of fecal samples was dissolved in $5 \mathrm{~mL}$ sterile saline, shaken well for $3 \mathrm{~min}$, and centrifuged at $4{ }^{\circ} \mathrm{C}$ for $3 \mathrm{~min}$, and the supernatant was collected and used as a transplant material. To reduce changes in the microbial population, fresh supernatants were prepared within 30 min prior to use. After 1 week of antibiotic treatment, the mice were given $150 \mu \mathrm{L}$ of the above supernatant by gavage twice at a 1-day interval [23].

\section{Tissue processing}

The mice were euthanized by $\mathrm{CO}_{2}$ asphyxiation, and the venous blood was collected. The cells of $\mathrm{CD} 45^{+}$, $\mathrm{CD}^{+} \mathrm{CD}^{+}$, and $\mathrm{CD} 3^{+} \mathrm{CD} 8^{+}$were measured by the Accurit ${ }^{\text {tm }}$ C6 Plus flow cytometer. After centrifugation, serum samples were obtained, and the content of sPD-L1 was measured by ELISA kits. The subcutaneous tumor was removed, and the long and short diameters of the tumor were measured with vernier calipers. Tumor volume was calculated by a vernier caliper. An electronic balance was used to weigh the tumor. Colonic contents were retained for 16SrRNA sequencing.

\section{Immunohistochemistry}

Fifty surgical specimens and the tumors of mice were fixed with formalin, treated with routine treatment, fixed with paraffin, and cut into $4 \mu \mathrm{m}$ sections for IHC. We used primary antibodies against PD-L1 (Bioss), CD8 (ab237709), and CD4 (ab133616, Bioss). A pathologist examined all the images. The number of $\mathrm{CD} 4^{+}$and $\mathrm{CD} 8^{+}$cells is expressed as an average of five random high-power fields (HPF). Intravascular positive cells were not counted. PD-L1 positive staining was defined as $>1 \%$ cellular reactivity.

\section{ELISA}

Serum specimens are stored in a refrigerator at $-80{ }^{\circ} \mathrm{C}$. According to the instructions of the ELISA kit(FANKEW), the optical density value of the final reaction product on the microplate analyzer was plotted at $450 \mathrm{~nm}$. Concentrations were calculated from absorbance readings on CD4, CD8, and SPD-L1 standard curves.

\section{Flow cytometry}

Antibody purchased from MULTISCIENCES: (CD45:70AR4505; CD3:70-AR00301; CD4:70-AR0040210; CD8:70-AR00804). Blood samples were divided into two groups and tested under the same conditions, one group for $\mathrm{CD} 45, \mathrm{CD} 3$, and $\mathrm{CD} 8$, and the other group for CD3 and CD4. Appropriate isotype controls were used when applicable. The Accuri ${ }^{\text {tm }}$ C6 Plus flow cytometer was used for detection.

\section{Statistical analysis}

The data analysis was performed using Prism 8.0 software (GraphPad), and analyzed by one-way ANOVA with Tukey's multiple comparisons test, or by paired or unpaired student's $t$ test as indicated. Data were considered to be significantly different at $P<0.05, P<0.01$, and $P<0.001$ represented in the figures and tables as $*, * *$, and $* * *$, respectively. Figures were processed using GraphPad Prism 8, Adobe illustrator CS5, and Adobe Photoshop CS4 software.

\section{Results}

\section{Characteristics of the intestinal flora}

To study the intestinal flora of patients with different types of PAs and those of healthy controls, fecal samples of 75 donors from three groups were analyzed using high-throughput sequencing targeting the $\mathrm{V} 3-\mathrm{V} 4$ region of the $16 \mathrm{~S}$ rRNA gene. As the sample size of each group increased, we found that species diversity increased and eventually plateaued, indicating that there was sufficient sampling for data analysis (Fig. 1a). The Shannon index indicated no significant difference in $\alpha$-diversity among the three groups (Fig. 1b). Thus, there was no significant difference in species richness and evenness of intestinal flora. We further investigated the differences in species between samples. Ordination of unweighted-UniFrac dissimilarity using principal coordinate analysis (PCoA) revealed separation of the three groups (Fig. 1c). The significant differences in $\beta$-diversity among the three groups of intestinal flora indicate that separation was associated with differences among community compositions (Fig. 1d). Furthermore, at the genus level, we observed differences in the intestinal flora between the three groups. Bacteroides, Biautia, Enterococcus, Megamonas, as well as other genera were differentially represented among the three groups (Fig. 1e). There generally were significant differences among the compositions of the intestinal flora of patients with NFPA, GHPA, and those of healthy controls. 

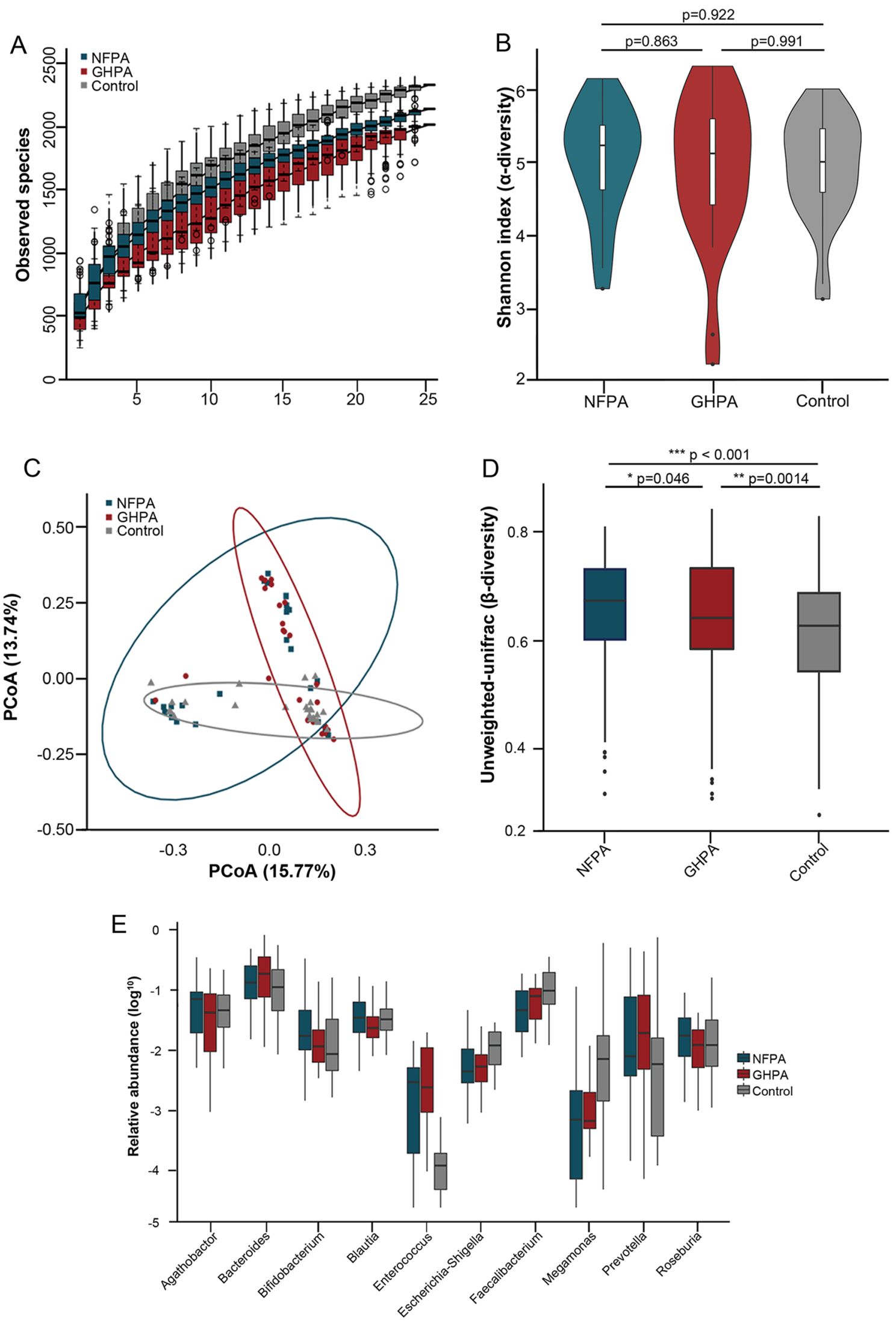
4Fig. 1 Characteristics of intestinal flora of the GHPA, NFPA, and healthy control groups. a Intestinal flora species accumulation of the GHPA $(n=25)$, NFPA $(n=25)$, and healthy groups $(n=25)$. b Fecal microbiome. The $\alpha$-diversities of fecal microbiomes were evaluated using Shannon index according to the number of OTUs. c Principal coordinate analysis of samples from the GHPA, NFPA, and control groups. Each symbol represents a sample. d The $\beta$-diversities among the three groups significantly differed among groups. e Top 10 genera with the greatest differences in relative abundance. Each box plot depicts the values of the median, dispersion, maximum, minimum, and outliers

\section{Prediction of the function of the intestinal flora}

We next evaluated species-level functions. For this purpose, we analyzed operational taxonomic units (OTUs) by generating a Venn diagram according to clustering. The numbers of OTUs specific to the GHPA, NFPA, and control groups were 314, 371, and 618, respectively (Fig. 2a). Functional analysis of intestinal flora in different groups indicated that the intestinal microflora in the three groups differentially affected the immune system (Fig. 2b). Overall, the influence of immune systems of the three groups of flora exhibited functional differences.

\section{Immune characteristics of PA}

To evaluate the activities of immune cells and PD-L1 expression in GHPA compared with NFPA, we simultaneously analyzed tumor and peripheral blood samples. Examples of IHC analysis of the numbers of $\mathrm{CD} 4^{+}$cells and $\mathrm{CD}^{+}$ cells and PD-L1 expression are shown in Fig. 3a. CD $4^{+}$cells and $\mathrm{CD}^{+}$cells were more frequent in GHPA than in NFPA samples (Fig. 3b, c), and the proportions of PD-L1-positive tumors in GHPA and NFPA samples were 64\% (16/25) and $4 \%(1 / 25)$, respectively (Fig. 3d). When we measured the levels of the three markers in serum samples from the corresponding patients and healthy controls, we found that the levels of CD4, CD8, and sPD-L1 in patients with GHPA were significantly higher compared with those in NFPA patients and healthy controls and that there was no significant difference between patients with NFPA and healthy controls (Fig. 3e-g). These data indicate that patients with
A

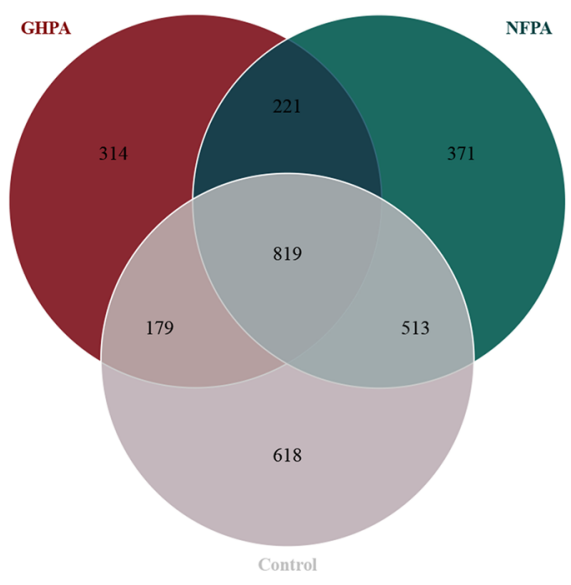

B

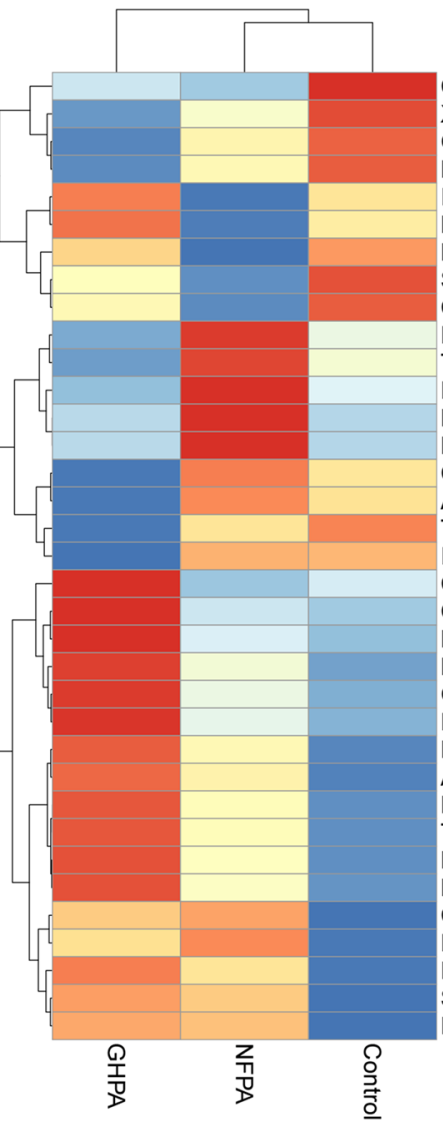

Cell_motility Xenobiotics biodegradation and metabolism Genetic_information_processing Membrane_transport mmune_system Metabolism

Metabolism of cofactors and vitamins Signal_transduction Cancers

Replication_and_repair Translation Endocrine and metabolic diseases Metabolism_of terpenoids and polyketides Nucleotide_metabolism Cellular_community__prokaryotes Amino acid_metabolism Transcription Infectious_diseases Cellular_processes_and_signaling Carbohydrate metabolism Biosynthesis_of_other_secondary_metabolites Metabolism_of_other_amino_acids Glycan_biosynthesis_and_metabolism Energy_metabolism Drug_resistance Aging

Poorly_characterized Transport_and_catabolism Lipid_metabolism Nervous system Cell_growth_and_death Endocrine_system Enzyme families Signaling_molecules and interaction Folding,_sorting_and_degradation

Fig. 2 Prediction of intestinal flora function. a Unique numbers of OTUs of the three groups. (b) Functional annotation of samples and heat-map clustering of abundance information 


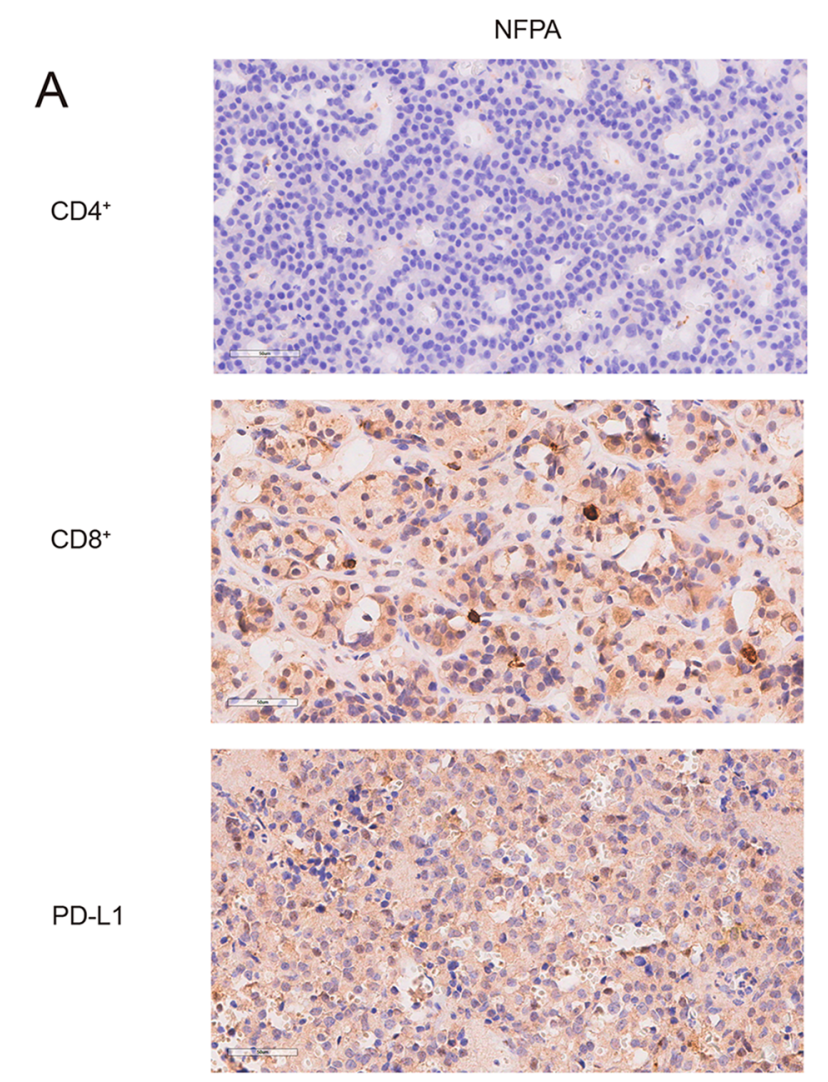

GHPA
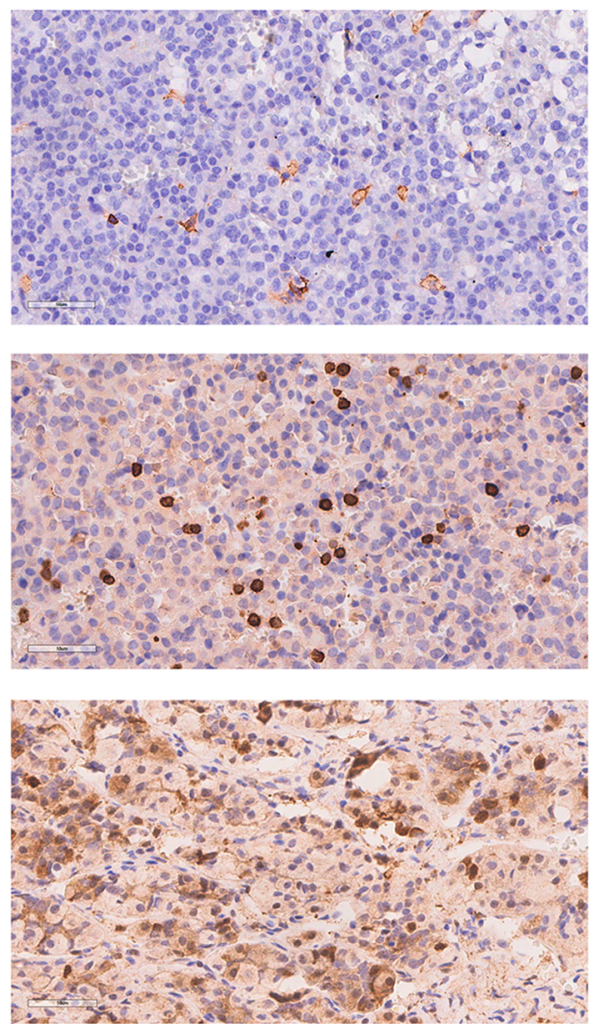

B

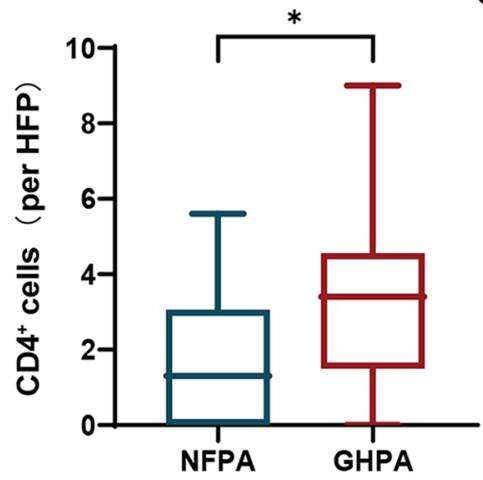

C

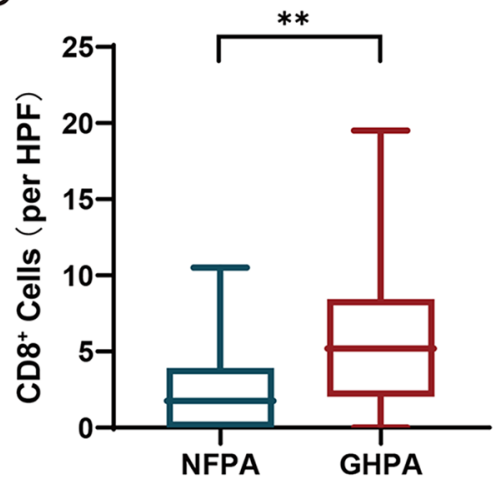

$E$

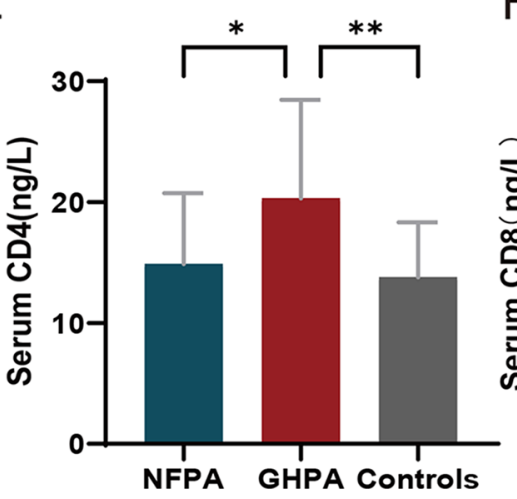

F

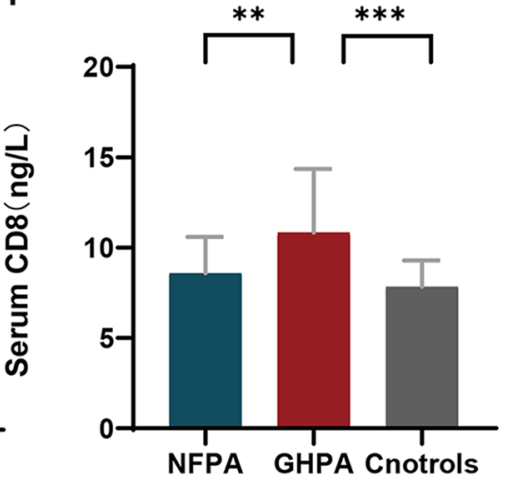

D
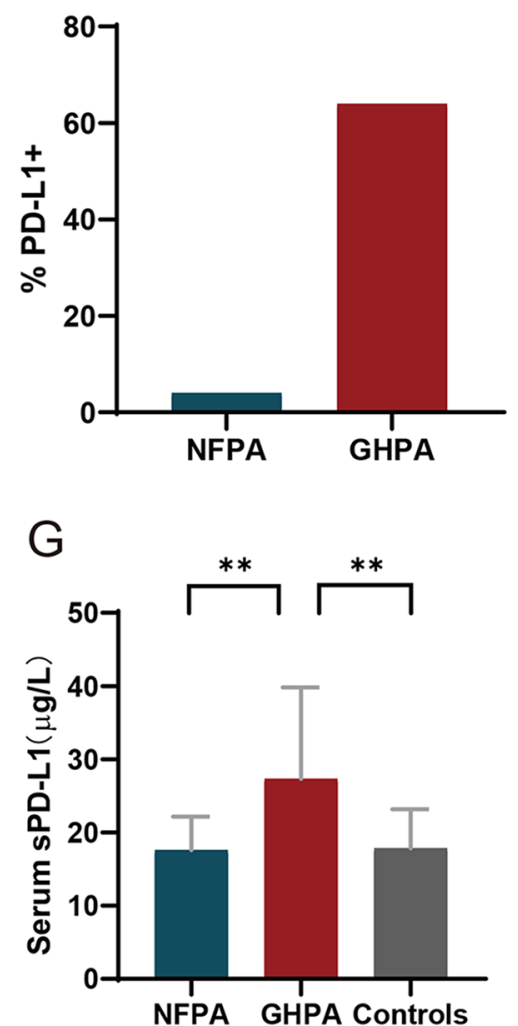
4 Fig. 3 IHC analysis of the expression of PD-L1 and the numbers of $\mathrm{CD} 4^{+}$cells and $\mathrm{CD} 8^{+}$cells in the GHPA $(n=25)$ and NFPA $(n=25)$ groups; and serum ELISA results. a Representative IHC data of GHPA and NFPA samples showing that PD-L1 levels and numbers of $\mathrm{CD}^{+}$cells and $\mathrm{CD} 4^{+}$cells were frequently higher in GHPA samples. PD-L1 positivity was defined as $>1 \%$ staining of cells. b, c Numbers of $\mathrm{CD}^{+}$cells in the GHPA group versus the NFPA group $(P=0.0197)$. Numbers of $\mathrm{CD} 8^{+}$cells in the GHPA and NFPA groups $(P=0.0086)$. d PD-L1-positivity rates in the GHPA group vs the NFPA group (64\% vs. $4 \%$ ). e-g The serum levels of CD4, CD8 and SPD-L1 in patients with GHPA were significantly higher compared with those of the other two groups

GHPA had a distinct immune environment, particularly that associated with the TIME.

\section{GHPA-FMT promotes tumor growth}

To determine the influence of the intestinal flora on the growth of tumors and immune functions, we established a subcutaneous tumor model of mice with immunodeficiency and injected PBMC extracted from the blood of Wistar rats through the tail vein to achieve immune reconstruction. The mice were then given broad-spectrum antibiotic (ATB) compounds for 1 week to deplete intestinal flora. Finally, 30 mice were divided into three groups with 10 mice in each group. The mice in the three groups were, respectively, gavaged with the fecal supernatant of two NFPA patients, two GHPA patients, and two control donors to realize FMT (Fig. 4a). The presence of T lymphocytes in peripheral blood was detected using flow cytometry after mice received reinfusion of PMBCs (Fig. 4b). To prove whether FMT regulated the intestinal microflora of the mice, we sequenced the $16 \mathrm{~S}$ rRNA (V3 + V4 regions) of the colon contents and found that the composition of the intestinal microflora of the three groups of mice differed and showed similar results in functional prediction as in human samples (Supplementary Fig. 1). The tumor phenotype is shown in (Fig. 4c). GHPAFMT significantly increased tumor weight and volume compared with the Control-FMT and NFPA-FMT groups (Fig. 4d, e). These data indicate that the growth of tumors in GHPA-FMT mice exceeded that of NFPA-FMT and ControlFMT mice.

\section{GHPA-FMT affects the systemic immune response}

To determine the influence of FMT on the immune system, we measured the levels of immune markers in tumors and peripheral blood. Representative IHC data for the expression of CD4, CD8, and PD-L1 are shown in Fig. 5a-c. In tumor tissues, GHPA-FMT increased the diffusely positive rate of PD-L1 ${ }^{+}$cells, and the number of PD-L1-positive cells was higher compared with those of the other two groups, which exhibited few PD-L1-positive cells (Fig. 5a). Furthermore, FMT promoted the infiltration of tumors by $\mathrm{CD}^{+}$cells, but not that of $\mathrm{CD}^{+}$cells (Fig. 5b, c). When we measured the proportion of $\mathrm{T}$ lymphocyte subsets in the blood (Fig. 5d), we found that GHPA-FMT increased the proportion of $\mathrm{CD}^{+}{ }^{+} \mathrm{CD}^{+}$cells in the blood (Fig. 5e). ELISAs revealed that GHPA-FMT increased the levels of sPD-L1 in peripheral blood (Fig. 5f). These results indicate that GHPA-FMT affected the changes of immune indexes in blood and the infiltration of tumors by immune cells.

\section{Discussion}

The richness and diversity of the types of intestinal flora can reflect a person's health [24]. For example, intestinal flora is an important factor in central nervous system disease, contributing to the maintenance of the host's immune homeostasis [14, 15]. However, there is no direct evidence of a relationship between intestinal flora and PAs. Here we identified significant differences in intestinal microbiota between patients with NFPA and those with GHPA, as well as healthy controls, and evaluated their effects on tumors using a mouse tumor xenograft model. Furthermore, to identify potential targets of the intestinal flora, we modified a mouse tumor model through immune reduction technology and found that after GHPA-FMT, the number of PD-L1-positive cells in tumor tissues increased, accompanied by increased infiltration of tumors by $\mathrm{CD}^{+}$cells, which was also reflected by the cognate lymphocyte subset in peripheral blood. Considering the complex relationship between PD-1 and PD-L1, we also analyzed the expression of PD-1 in tumor tissues, while no significant difference was observed (Supplementary Fig. 2). Our findings reveal potential mechanisms through which the intestinal flora affects PA (Fig. 6).

PD-L1 is expressed by numerous cell types, and its expression on the surface of tumor cells is a driving factor for tumor growth as the tumor escapes from the pursuit of immune cells [25]. The binding of PD-1 to PD-L1 alters the activity of $T$ cells through numerous mechanisms, thereby inhibiting $T$ cell proliferation, survival, and their ensuing effects [25]. Moreover, the level of infiltration of T cells in GHPA and the expression of PD-L1 are higher compared with other PAs, which is consistent with the results of our present study [8, 26-28]. We show here that the populations of $\mathrm{CD}^{+}{ }^{+}$cells, $\mathrm{CD} 8^{+}$cells, and the levels of PD-L1 in GHPA were higher compared with those of NFPA, indicating a unique association of the TIME with GHPA. However, some reports indicate that the sizes of the populations of $\mathrm{CD}^{+}{ }^{+}$cells did not significantly differ between GHPA and NFPA [29]. This may be explained by differences in sample selection and the relatively small sample size that must be increased in future research. The larger populations of $\mathrm{CD}^{+}$ cells and $\mathrm{CD}^{+}$cells and increased serum levels of sPD-L1 in patients' peripheral blood with GHPA suggest that the 

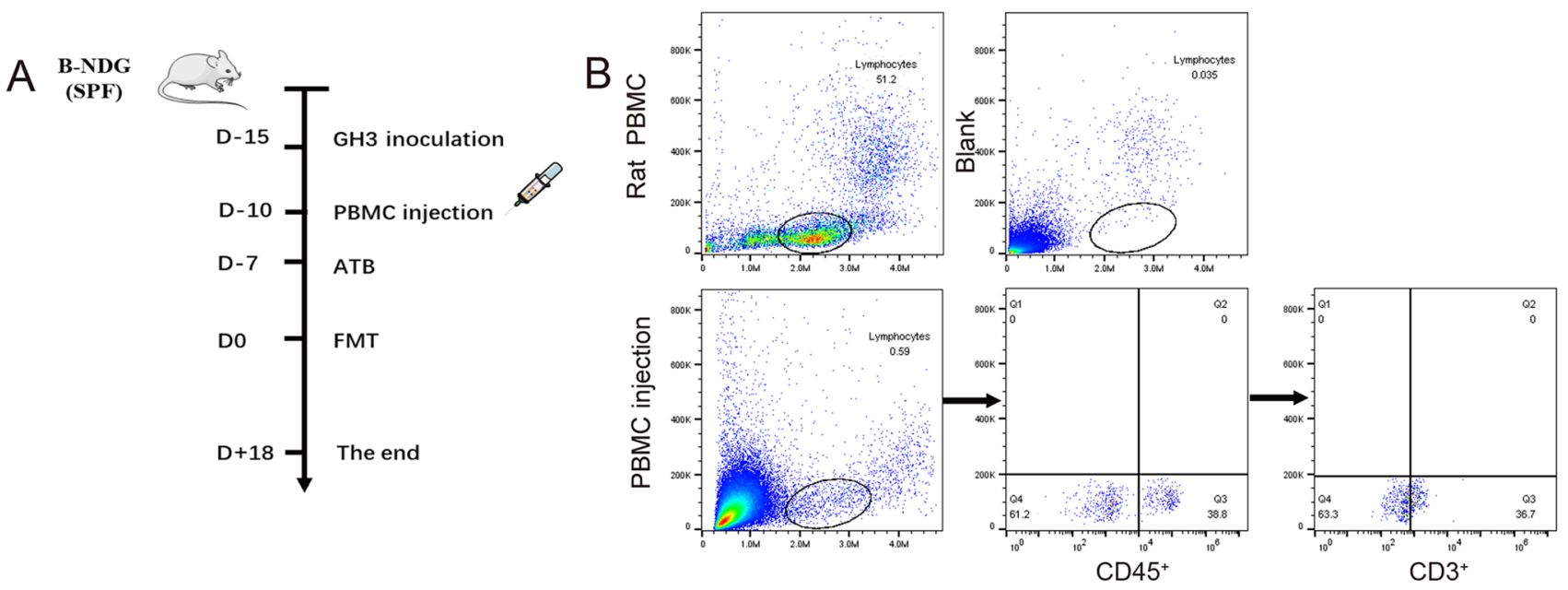

\section{C GHPA-FMT \\ NFPA-FMT \\ Control-FMT

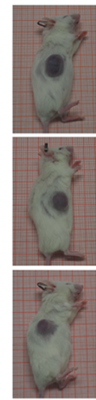

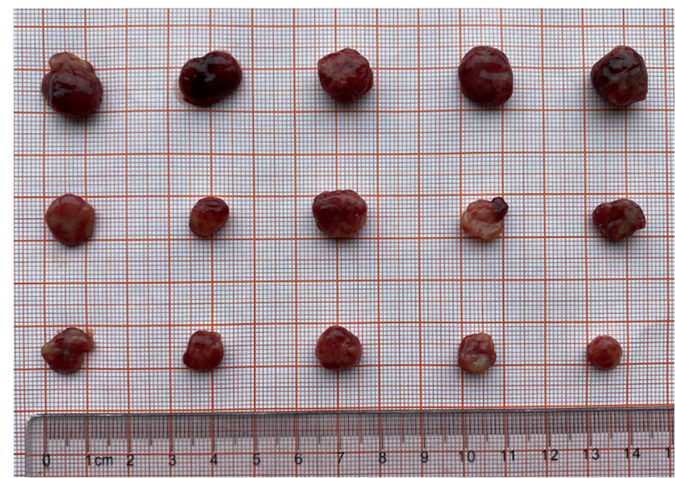
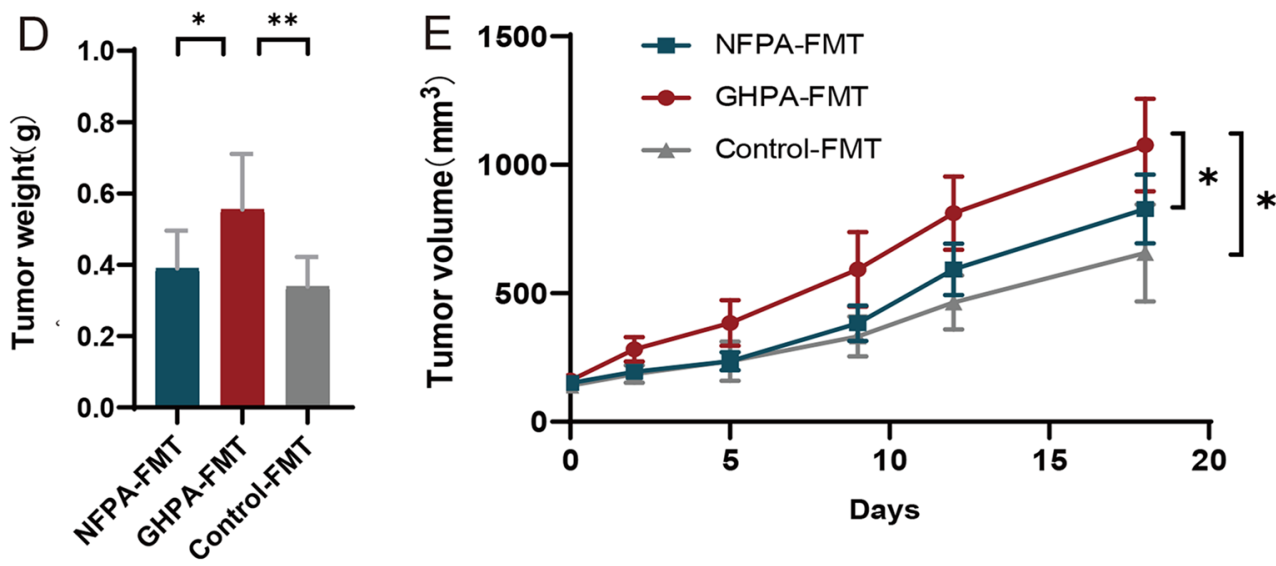

Fig. 4 GHPA-FMT promotes tumor growth. a Experimental scheme. b Representative flow cytometry analysis. After adoptive transfer of PBMCs, lymphocytes, $\mathrm{CD} 45^{+}$cells, and $\mathrm{CD}^{+}$cells were detected in the peripheral blood of mice. There were no lymphocytes in the control group. c-e Images of the solid tumor (c), tumor weights (d), and tumor volumes (e) tumor not only affects the TIME but also profoundly exerts systemic effects involving immunity.

A stable microbial flora structure and human health are mutually reinforcing, and changes in one will lead to a corresponding response in the other. For the intestinal flora, the host influences the adaptability of individual bacteria in a competitive environment, and therefore the suboptimal function of the microbiota degrades the health of the host [30]. Here we found no significant difference in the $\alpha$-diversity index (richness and diversity) of intestinal flora between the NFPA, GHPA, and control groups. However, there were significant differences in the $\beta$-diversity index (both structure 

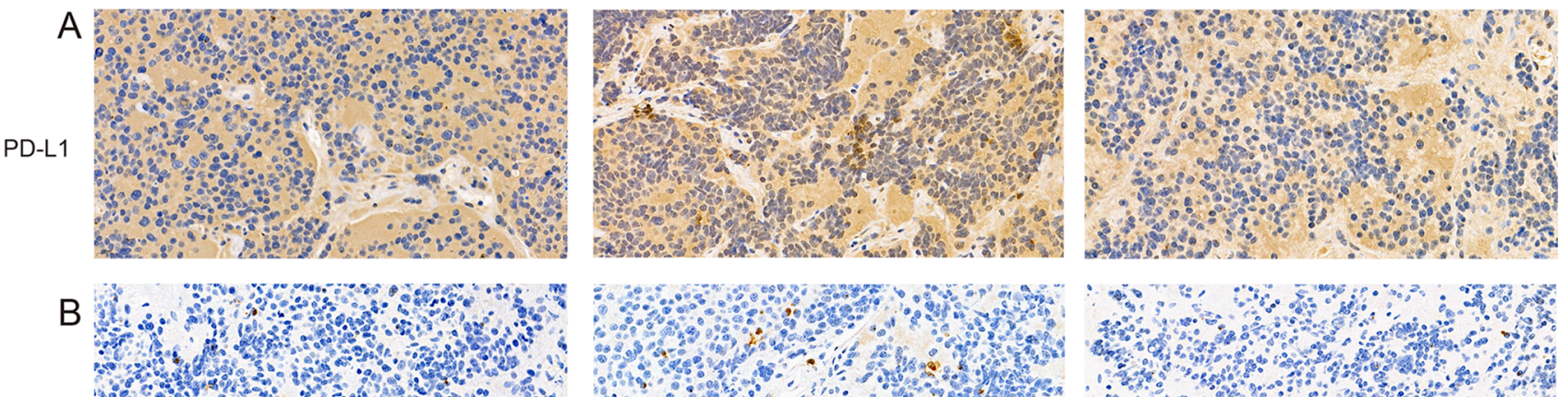

$\mathrm{CD}^{+}$

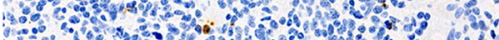

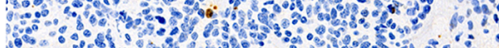
10. \%
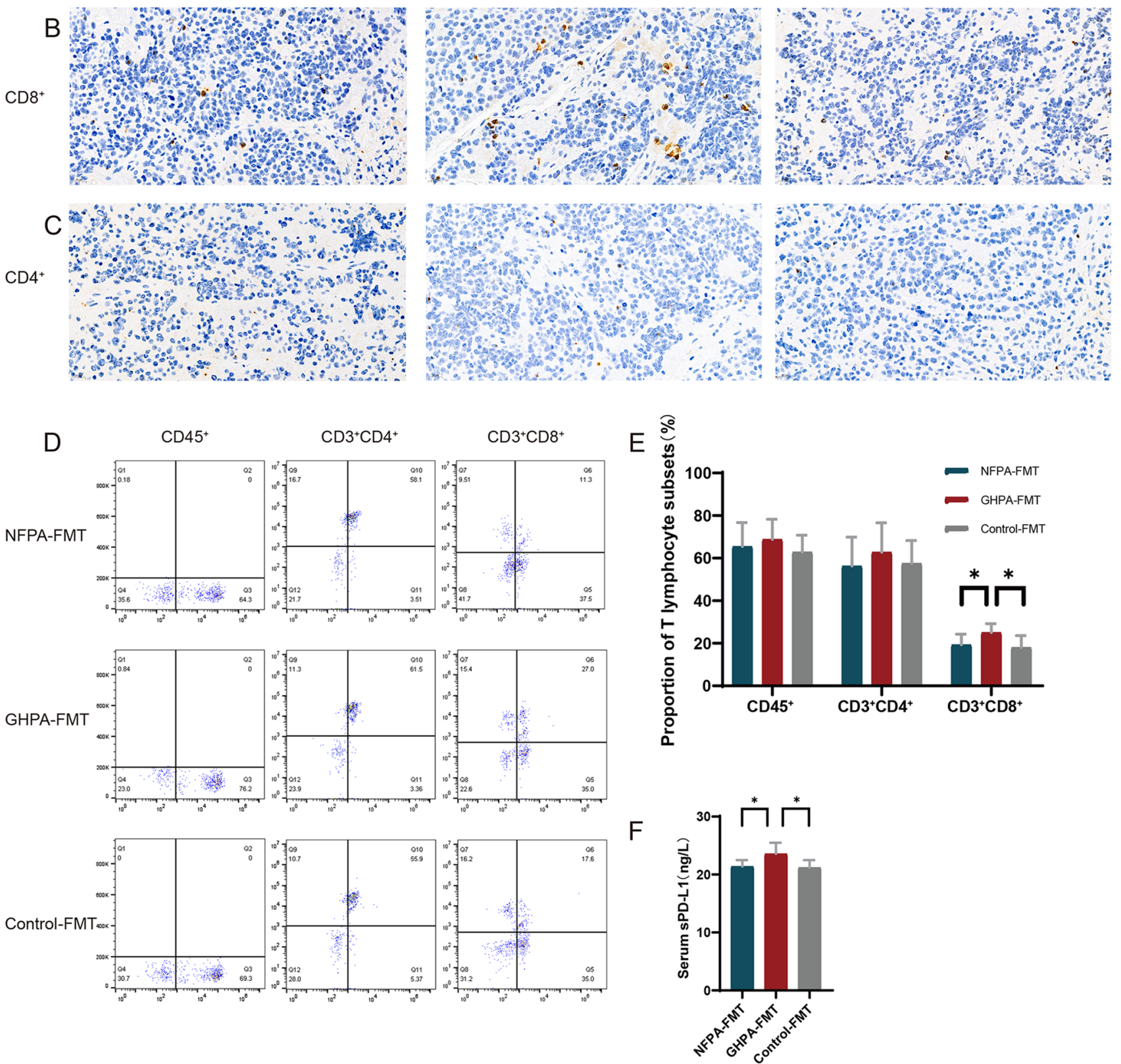

Fig. 5 GHPA-FMT promotes the expression of PD-L1 and the infiltration of $\mathrm{CD}^{+}$cells in PAs and affects their numbers in peripheral blood. a-c Representative IHC analysis. Compared with NFPA-FMT and control-FMT groups, the GHPA-FMT group comprised significantly increased the numbers of PD-L1-positive cells and infiltration of $\mathrm{CD}^{+}$cells, although there was no significant difference in the number of $\mathrm{CD}^{+}$cells. d, e Representative flow cytometry analysis. GHPA-FMT significantly increased the proportion of $\mathrm{CD}^{+} \mathrm{CD}^{+}$ cells in peripheral blood. f Serum ELISA. GHPA-FMT significantly increased the levels of SPD-L1 


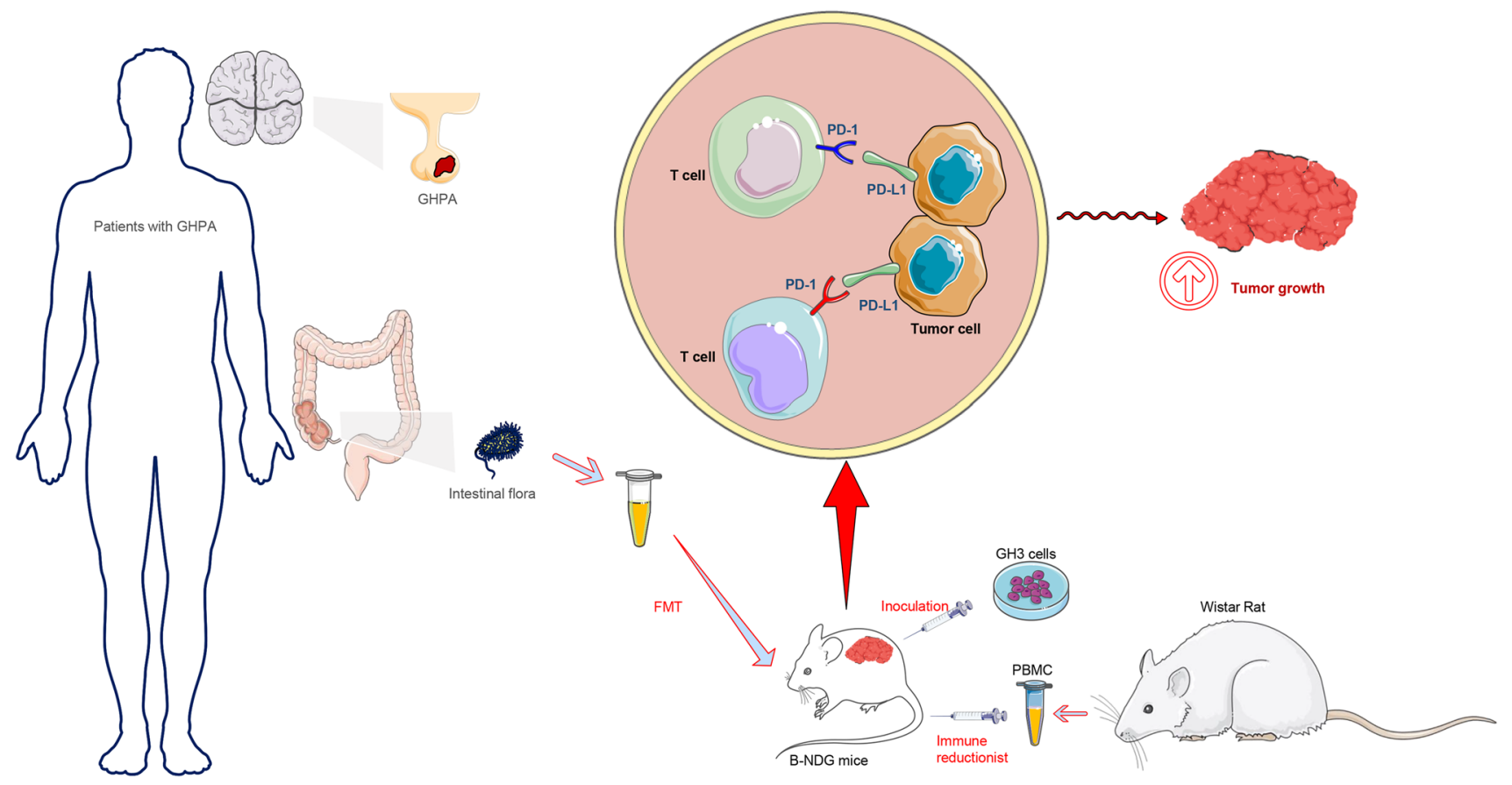

Fig. 6 Experimental protocol. In B-NDG mice after immune reduction and tumor inoculation, PD-L1 levels in tumor tissues increased after GHPA-FMT, and tumor growth subsequently increased

and quantity) between the three groups, indicating that the composition of the intestinal microbiota of each significantly differed. This difference might be caused by a variety of factors, including diet, age, sex, hormone levels, and disease status [31]. GH and insulin-like growth factor 1 (IGF-1) in patients with GHPA are significantly higher than those with NFPA and normal controls [32]. Recently, researchers have found that the gut microbiota of male mice changes in response to excessive levels of GH [33]. In addition, gut microbiota can influence host hormone levels, such as auxinreleasing peptide and cortisol, suggesting a complex interaction between the GH/IGF-1 axis and intestinal flora [34, 35]. However, there were also differences in the composition of gut microbiota between the NFPA group and normal control, while no differences in hormone levels between the two groups. Thus, we speculate that the reasons for the differences in intestinal flora composition among the three groups in this study are complex and diverse, not limited to the hormone levels, which needs further study.

Robust data indicate that the composition of the intestinal flora is a major environmental factor that varies between individuals and influences systemic immunity [36]. For example, tumors in mice that receive intestinal flora from patients with metastatic melanoma sensitive to anti-PD-1 therapy have higher $\mathrm{CD}^{+} T$ cell densities and higher levels of PD-L1 compared with those of the nonsensitive group [37]. Furthermore, antibiotic treatment of altered intestinal microflora in mice with gliomas changes the distribution of
NK cell subsets and decreases antitumor effects, suggesting that the alteration of the intestinal flora may affect the growth of brain tumors by affecting the immune response [38]. Here we found that the number of PD-L1-positive cells in the tumors of GHPA-FMT mice was significantly higher compared with the NFPA-FMT and Control-FMT groups, which may represent an important factor leading to increased tumor growth in GHPA-FMT mice. Thus, GHPA-FMT caused an increase in immune escape from tumors. The factors affecting PD-L1 expression in tumors seem to be quite complex, including epigenetic changes, signaling pathways, and transcriptional regulation, cytokines and oxidative stress, etc. [39-41]. Robust data reveal that gut microbes can influence these factors. Bacteroides (one of the different species found in this study), for example, can convert cellulose, lignin, and pectin into short-chain fatty acids and subsequently affect the disease of the central nervous system through oxidative stress [42]. Intestinal flora can regulate IFN- $\gamma$ production, and Freeman et al. demonstrated that IFN $\gamma$ upregulates PD-L1 in monocytes and dendritic cells [43, 44]. We thus hypothesize that the gut microbiota could regulate PD-L1 expression of pituitary adenoma via regulation of metabolism and release of cytokines, and we will validate the hypothesis in our future work. Interestingly, a recent study indicated that the infiltration of $\mathrm{CD}^{+} \mathrm{T}$ cells in the tumor was significantly positively correlated with the abundance of Faecalibacterium, Ruminococcaceae, and Clostridiales 
and negatively correlated with Bacteroidales(though not significant) [45], revealing intestinal flora may influence $T$ cells infiltrating in tumors. Moreover, in colorectal cancer, intestinal microbiome-derived stimulation induces chemokine expression in tumor cells, ultimately enhancing $T$ cell recruitment into tumor [46]. Our results showed more infiltration of $\mathrm{CD}^{+}$cells and higher PD-L1 expression in GHPA-FMT mice compared to the NFPA-FMT and control-FMT mice, while larger tumors were observed in the GHPA-FMT mice. We believe the limited increase in $\mathrm{CD}^{+}$ cells in the GHPA-FMT mice may be explained not only by the changes in intestinal flora but also by compensation for immune escape after the increased PD-L1expression. When the function of immune cells is suppressed, the body's immune system increases their numbers. Further research is required to prove this hypothesis. Moreover, there is evidence that the presence of $\mathrm{CD} 8^{+}$cells in tumors predicts the clinical response to anti-PD-1 therapy, and the increase in these cells after GHPA-FMT provides a basis for further exploration of GHPA-specific immunotherapy. In contrast, a similar result has not obtained for NFPA-FMT and Control-FMT mice, suggesting that differences in the intestinal flora between patients with GHPA and these latter groups may cause changes in the tumor microenvironment, thereby affecting tumor growth. Moreover, the increased proportion of $\mathrm{CD}^{+} \mathrm{CD}^{+}$cells in peripheral blood and the elevated levels of sPD-L1 suggest that GHPA-FMT systemically affects the immune system.

There are several limitations to our study. First, we studied a mouse model of PA with the technical limitations of direct cellular implantation, which does not perfectly reproduce the anatomy of PA, although we believe we convincingly demonstrate the influence of intestinal flora on PA in vivo. Furthermore, the mouse model is relatively mature and stable as established by related studies of PA; and we provide proof of concept of the influence of intestinal flora on PA. Second, we used immune reconstruction technology to simulate the immune environment in animals, which is insufficient for comprehensive immune research. Moreover, highly complex factors affect tumor growth, immunity is only one of them. Finally, the mechanism through which intestinal flora influences tumor growth through immune response remains to be further explored, requiring a larger sample size and cell-level studies, which are our future research directions.

\section{Conclusion}

The different compositions and distributions of the intestinal flora of patients with PA exert systemic effects on the immune system. GHPA-FMT promotes the growth of
PA, which may be achieved through increasing the number of PD-L1-positive cells in tumors, thereby enhancing the escape of tumor cells from the immune response. There may be a compensatory increase in the infiltration rate of $\mathrm{CD}^{+}{ }^{+}$cells when their cytotoxic function is inhibited.

Supplementary Information The online version contains supplementary material available at https://doi.org/10.1007/s00262-021-03080-6.

Acknowledgements The authors thank all the patients who trusted them and all the physicians and staff who helped them in this study.

Author contributions All authors contributed to the study conception and design. Material preparation, data collection, and analysis were performed by DN, QF, BL, and ML. The first draft of the manuscript was written by DN. The manuscript was modified by HW, CL, SG, and YZ. PZ finally reviewed the manuscript and all authors commented on previous versions of the manuscript. All authors read and approved the final manuscript.

Funding No funding was received for conducting this study.

Data availability Some or all datasets generated during and analyzed during the current study are not publicly available but are available from the corresponding author on reasonable request.

\section{Declarations}

Conflict of interest The authors declare that they have no competing interest.

Ethical approval All studies were conducted under the approval of the Institutional Review Committee of Beijing Tiantan Hospital, Capital Medical University, and the study was conducted by the Principles of Good Clinical Practice and the Declaration of Helsinki.

Consent to participate Informed consent was obtained from all individual participants included in the study.

Consent for publication Patients signed informed consent regarding publishing their data and photographs.

Open Access This article is licensed under a Creative Commons Attribution 4.0 International License, which permits use, sharing, adaptation, distribution and reproduction in any medium or format, as long as you give appropriate credit to the original author(s) and the source, provide a link to the Creative Commons licence, and indicate if changes were made. The images or other third party material in this article are included in the article's Creative Commons licence, unless indicated otherwise in a credit line to the material. If material is not included in the article's Creative Commons licence and your intended use is not permitted by statutory regulation or exceeds the permitted use, you will need to obtain permission directly from the copyright holder. To view a copy of this licence, visit http://creativecommons.org/licenses/by/4.0/. 


\section{References}

1. Aflorei E, Korbonits M (2014) Epidemiology and etiopathogenesis of pituitary adenomas. J Neurooncol 117(3):379-394

2. Shi C, Ye Z, Han J et al (2020) BRD4 as a therapeutic target for nonfunctioning and growth hormone pituitary adenoma. Neuro Oncol 22(8):1114-1125

3. Takamizawa T, Horiguchi K, Nakajima Y et al (2019) Central hypothyroidism related to pituitary adenomas: low incidence of central hypothyroidism in patients with acromegaly. J Clin Endocrinol Metab 104(10):4879-4888

4. Gerges MM, Rumalla K, Godil SS, Younus I, Elshamy W, Dobri GA, Kacker A, Tabaee A, Anand VK, Schwartz TH (2020) Long-term outcomes after endoscopic endonasal surgery for nonfunctioning pituitary macroadenomas. J Neurosurg. 134(2):1-12. https://doi.org/10.3171/2019.11.JNS192457

5. Cardinal T, Rutkowski MJ, Micko A et al (2020) Impact of tumor characteristics and pre- and postoperative hormone levels on hormonal remission following endoscopic transsphenoidal surgery in patients with acromegaly. Neurosurg Focus 48(6):E10

6. Buchta Rosean CM, Rutkowski MR (2017) The influence of the commensal microbiota on distal tumor-promoting inflammation. Semin Immunol 32:62-73

7. Mei Y, Bi WL, Greenwald NF et al (2016) Increased expression of programmed death ligand 1 (PD-L1) in human pituitary tumors. Oncotarget 7(47):76565-76576

8. Wang PF, Wang TJ, Yang YK et al (2018) The expression profile of PD-L1 and CD8(+) lymphocyte in pituitary adenomas indicating for immunotherapy. J Neurooncol 139(1):89-95

9. Sato M, Tamura R, Tamura H et al (2019) Analysis of tumor angiogenesis and immune microenvironment in non-functional pituitary endocrine tumors. J Clin Med. 8(5):595

10. Kemeny HR, Elsamadicy AA, Farber SH et al (2020) Targeting PD-L1 initiates effective antitumor immunity in a murine model of cushing disease. Clin Cancer Res 26(5):1141-1151

11. Tsushima F, Yao S, Shin $T$ et al (2007) Interaction between B7-H1 and PD-1 determines initiation and reversal of T-cell anergy. Blood 110(1):180-185

12. Taube J, Anders R, Young G et al (2012) Colocalization of inflammatory response with B7-h1 expression in human melanocytic lesions supports an adaptive resistance mechanism of immune escape. Science Transl Med 4(127):127ra137

13. Pardoll DM (2012) The blockade of immune checkpoints in cancer immunotherapy. Nat Rev Cancer 12(4):252-264

14. Sharon G, Sampson TR, Geschwind DH, Mazmanian SK (2016) The central nervous system and the gut microbiome. Cell 167(4):915-932

15. Hoban AE, Stilling RM, Ryan FJ et al (2016) Regulation of prefrontal cortex myelination by the microbiota. Transl Psychiatry 6(4):e774

16. Brown JM, Hazen SL (2018) Microbial modulation of cardiovascular disease. Nat Rev Microbiol 16(3):171-181

17. Han B, Sivaramakrishnan P, Lin C-CJ et al (2017) Microbial genetic composition tunes host longevity. Cell 169(7):1249-1262

18. Tanoue T, Morita S, Plichta D et al (2019) A defined commensal consortium elicits CD8 T cells and anti-cancer immunity. Nature 565(7741):600-605

19. Sivan A, Corrales L, Hubert $\mathrm{N}$ et al (2015) Commensal Bifidobacterium promotes antitumor immunity and facilitates anti-PD-L1 efficacy. Science 350(6264):1084-1089

20. Fukasaku Y, Goto R, Ganchiku Y et al (2020) Novel immunological approach to asses donor reactivity of transplant recipients using a humanized mouse model. Human Immunol 81(7):342-353
21. Issa F, Hester J, Goto R, Nadig SN, Goodacre TE, Wood K (2010) Ex vivo-expanded human regulatory $\mathrm{T}$ cells prevent the rejection of skin allografts in a humanized mouse model. Transplantation 90(12):1321-1327

22. Klicznik MM, Benedetti A, Gail LM et al (2020) A novel humanized mouse model to study the function of human cutaneous memory T cells in vivo in human skin. Sci Rep 10(1):11164

23. Borody TJ, Paramsothy S, Agrawal G (2013) Fecal microbiota transplantation: indications, methods, evidence, and future directions. Curr Gastroenterol Rep 15(8):337

24. Hollister EB, Gao C, Versalovic J (2014) Compositional and functional features of the gastrointestinal microbiome and their effects on human health. Gastroenterology 146(6):1449-1458

25. Sun C, Mezzadra R, Schumacher TN (2018) Regulation and function of the PD-L1 checkpoint. Immunity 48(3):434-452

26. Principe M, Chanal M, Ilie MD et al (2020) Immune landscape of pituitary tumors reveals association between macrophages and gonadotroph tumor invasion. J Clin Endocrinol Metab. https://doi. org/10.1210/clinem/dgaa520

27. Marques P, Barry S, Carlsen E et al (2020) The role of the tumour microenvironment in the angiogenesis of pituitary tumours. Endocrine 70(3):593-606

28. Zhao G, Chen W, He J et al (2020) Analysis of cyclooxygenase 2 , programmed cell death ligand 1 , and arginase 1 expression in human pituitary adenoma. World Neurosurg 144:e660-e673

29. Zhou W, Zhang C, Zhang D et al (2020) Comprehensive analysis of the immunological landscape of pituitary adenomas: implications of immunotherapy for pituitary adenomas. J Neurooncol 149(3):473-487

30. Backhed F, Ley RE, Sonnenburg JL, Peterson DA, Gordon JI (2005) Host-bacterial mutualism in the human intestine. Science 307(5717):1915-1920

31. Human Microbiome Project C. (2012) Structure function and diversity of the healthy human microbiome. Nature 486(7402):207-214

32. Schwarzer M, Strigini M, Leulier F (2018) Gut microbiota and host juvenile growth. Calcif Tissue Int 102(4):387-405

33. Jensen EA, Young JA, Jackson Z et al (2020) Growth hormone deficiency and excess alter the gut microbiome in adult male mice. Endocrinology. https://doi.org/10.1210/endocr/bqaa026

34. Yan J, Herzog JW, Tsang K et al (2016) Gut microbiota induce IGF-1 and promote bone formation and growth. Proc Natl Acad Sci USA 113(47):E7554-E7563

35. Clarke G, Stilling RM, Kennedy PJ, Stanton C, Cryan JF, Dinan TG (2014) Minireview: gut microbiota: the neglected endocrine organ. Mol Endocrinol (Baltimore, MD.) 28(8):1221-1238

36. Spranger S, Sivan A, Corrales L, Gajewski TF (2016) Tumor and host factors controlling antitumor immunity and efficacy of cancer immunotherapy. Adv Immunol 130:75-93

37. Gopalakrishnan V, Spencer CN, Nezi L et al (2018) Gut microbiome modulates response to anti-PD-1 immunotherapy in melanoma patients. Science 359(6371):97-103

38. D'Alessandro G, Antonangeli F, Marrocco F et al (2020) Gut microbiota alterations affect glioma growth and innate immune cells involved in tumor immunosurveillance in mice. Eur J Immunol 50(5):705-711

39. Glorieux C, Xia X, Huang P (2021) The role of oncogenes and redox signaling in the regulation of PD-L1 in cancer. Cancers 13(17):4426

40. Cretella D, Digiacomo G, Giovannetti E, Cavazzoni A (2019) PTEN alterations as a potential mechanism for tumor cell escape from PD-1/PD-L1 inhibition. Cancers 11(9): 1318

41. Cha JH, Yang WH, Xia W et al (2018) Metformin promotes antitumor immunity via endoplasmic-reticulum-associated degradation of PD-L1. Mol Cell. 71(4):606-620 
42. Takewaki D, Yamamura T (2021) Gut microbiome research in multiple sclerosis. Neurosci Res 168:28-31

43. Freeman GJ, Long AJ, Iwai Y et al (2000) Engagement of the PD-1 immunoinhibitory receptor by a novel B7 family member leads to negative regulation of lymphocyte activation. J Exp Med 192(7):1027-1034

44. Ding W, You Z, Chen Q et al (2021) Gut microbiota influences neuropathic pain through modulating proinflammatory and antiinflammatory T cells. Anesth Analg 132(4):1146-1155

45. Gopalakrishnan V, Spencer CN, Nezi L et al (2018) Gut microbiome modulates response to anti-PD-1 immunotherapy in melanoma patients. Science (New York, N.Y.) 359(6371):97-103
46. Cremonesi E, Governa V, Garzon JFG et al (2018) Gut microbiota modulate $\mathrm{T}$ cell trafficking into human colorectal cancer. Gut 67(11):1984-1994

Publisher's Note Springer Nature remains neutral with regard to jurisdictional claims in published maps and institutional affiliations. 